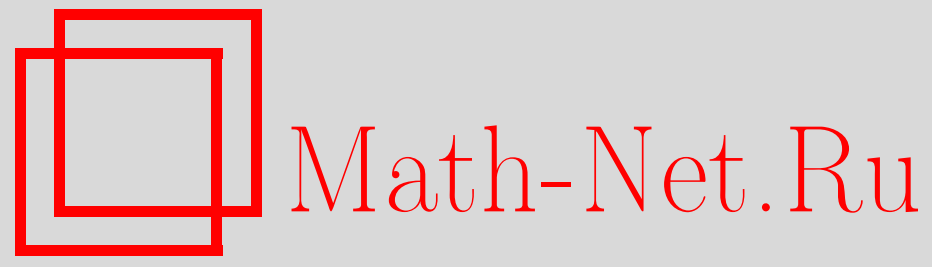

А. Г. Хованский, Семейства сечений квадрик и классические геометрии, Функи. анализ и его прил., 2000, том 34, выпуск 3, 91-95

DOI: https://doi.org/10.4213/faa321

Использование Общероссийского математического портала MathNet.Ru подразумевает, что вы прочитали и согласны с пользовательским соглашением

http://www. mathnet.ru/rus/agreement

Параметры загрузки:

IP : 54.162 .85 .209

26 апреля 2023 г., 12:01:58

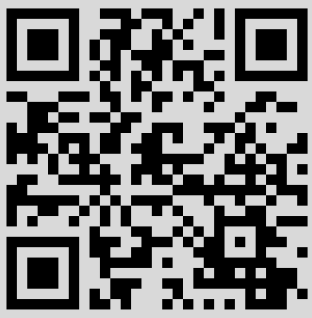




\title{
Семейства сечений квадрик и классические геометрии*
}

\author{
(c) 2000. А. Г. ХовАнский
}

Памяти моего отиа

Георгия Сергеевича Хованского

В заметке описываются все римановы метрики на невырожденных квадриках, геодезические относительно которых являются плоскими кривыми. Показывается, что все такие метрики имеют постоянную кривизну, а следовательно, локально задают на квадрике одну из классических геометрий. Настоящая заметка является непосредственным продолжением статьи [5], в которой решалась возникшая из практики задача о спрямлении окружностей. Эта практическая задача была поставлена Г. С. Хованским в связи с его работами о преобразовании номограмм из выравненных точек в циркульные номограммы [1-4].

1. Квадрические точки проективной поверхности. Скажем, что точка $A$ является квадрической точкой на ростке вещественной регулярной поверхности в вещественном проективном пространстве, если существует невырожденная квадрика, которая аномально близко приближает поверхность в этой точке, т.е. расстояние от точки $B$ на поверхности до квадрики является величиной четвертого порядка малости относительно расстояния от $A$ до $B$.

Аффинную систему координат $x, y, z$ в аффинной окрестности проективного пространства назовем приспособленной к поверхности в точке $A$, если начало координат совпадает с этой точкой, а плоскость $z=0$ касается рассматриваемой поверхности в точке $A$. Пусть локальное уравнение поверхности в окрестности точки $A$ в некоторой приспособленной системе координат имеет вид $z=f(x, y)=B_{2}(x, y)+K_{3}(x, y)+\ldots$, где $B_{2}$ и $K_{3}$ - соответственно квадратный и кубический члены ряда Тейлора функции $f$ в начале координат.

Лемма 1. (1) Точка $A$ является квадрической точкой поверхности, если $и$ только если кубический полином $K_{3}$ делится на полином второй степени $B_{2}$.

(2) Условие делимости полинома $K_{3}$ на полином $B_{2}$ является проективно инвариантньм, т. е. не зависит от выбора приспособленной системь координат.

(3) Точка $А$ является квадрической точкой поверхности, если и только если существует приспособленная система координат, для которой полином $K_{3}$ тождественно равен нулю.

ДокАЗАтЕЛЬСтво. (1) Пусть $K_{3}=L_{1} B_{2}$, где $L_{1}$ - однородный линейный полином от $x, y$. Тогда поверхность аномально близко приближается квадрикой $z=B_{2}+L_{1} z$. Обратно, всякая квадрика, касающаяся в нуле плоскости $z=0$, задается уравнением вида $z=B_{2}+L_{1} z+c z^{2}$, где $B_{2}$ и $L_{1}$ - однородные полиномы от $x, y$ второй и первой степени, а $c$ - константа. Решая это уравнение с точностью до кубических членов, получаем $z=B_{2}+L_{1} B_{2}+\ldots$ Пункт $(2)$ немедленно вытекает из п. (1). Утверждение в п. (3) в одну сторону тоже сразу вытекает из п. (1). Для доказательства в другую сторону достаточно выбрать

*Работа выполнена при частичной поддержке гранта 99-01-00245 Российского фонда фундаментальных исследований и Канадского гранта 156833-98. 
приспособленную систему координат, удовлетворяющую следующим дополнительным условиям: бесконечно удаленная плоскость совпадает с касательной плоскостью приближающей квадрики в некоторой точке квадрики, отличной от точки $A$, а ось $z$ проходит через эту бесконечно удаленную точку. В таких координатах приближающая квадрика задается уравнением $z=B_{2}(x, y)$, a полином $K_{3}$ обращается в нуль.

Невырожденная квадрика пересекается со своей касательной плоскостью по паре прямых, вещественных или комплексных. Несложно проверяется следующая

Лемма 2. Точка А является квадрической на ростке строго гиперболической поверхности, если и только если каждая из двух веток пересечения поверхности с касательной плоскостью в точке $A$ имеет перегиб в этой точке. Точка А является квадрической на ростке строго эллиптической поверхности, если и только если каждая из двух комплексньх веток пересечения комплексификации 3-струи поверхности с комплексной касательной плоскостью в точке $A$ имеет перегиб в этой точке.

ЗАмЕчАниЕ. Определение квадрической точки автоматически переносится на комплексные поверхности в комплексном проективном пространстве. Мне представляется интересной следующая задача: сколько квадрических точек имеет общая поверхность степени $n$ в комплексном проективном пространстве?

2. Спрямляемые семейства плоских сечений. Рассмотрим в окрестности точки нуль на плоскости росток гладкой функции $F$, разложение которой в ряд Тейлора с точностью до третьего порядка имеет вид $F(x, y)=$ $\mu x-y+P_{2}(x, y)+P_{3}(x, y)+\ldots$, где $\mu$ - число, а $P_{2}$ и $P_{3}$ - однородные полиномы соответственно степени 2 и 3 . Следующая лемма 3 проверяется прямым вычислением.

Лемма 3. Решение $y(x)$ уравнения $F(x, y(x))=0$ с точностью до третьего порядка малости задается формулой

$$
y(x)=\mu x+P_{2}(1, \mu) x^{2}+\left[\frac{\partial P_{2}}{\partial y}(1, \mu) P_{2}(1, \mu)+P_{3}(1, \mu)\right] x^{3}+\ldots
$$

СлЕДСТВИЕ 1. Пересечение поверхности $z=f(x, y)=B_{2}(x, y)+K_{3}(x, y)+\ldots$ с плоскостью $y=\mu x+a z$ в локальных координатах $x, y$ на поверхности $c$ точностью до третьего порядка малости задается формулой

$$
y(x)=\mu x+a B_{2}(1, \mu) x^{2}+\left[a^{2} \frac{\partial B_{2}}{\partial y}(1, \mu) B_{2}(1, \mu)+a K_{3}(1, \mu)\right] x^{3}+\ldots
$$

Следствие получается применением леммы 3 к уравнению $y=\mu x+a f(x, y)$.

Множество кривых на поверхности называется (локально) спрямляемыл, если существует (локальный) диффеоморфизм поверхности на плоскую область, переводящий каждую кривую из этого множества в интервал прямой линии. Пучком кривых с центром в точке $A$ назовем любое множество кривых на поверхности, проходящих через точку $A$. Если пучок кривых локально спрямляется около своего центра, то различные кривые из него имеют в центре пучка различные касательные. Рассмотрим пучок кривых на плоскости с центром в начале координат и обозначим через $\gamma_{\mu}$ кривую из пучка, которая в начале координат касается прямой $y=\mu x$. В окрестности начала координат кривая $\gamma_{\mu}$ является графиком некоторой функции $y_{\mu}(x)$. 
ЛЕмма 4. Если пучок кривьх $\left\{y=y_{\mu}(x)\right\}$ локально спрямляется около точки нуль, то существуют некоторье полиномь $T_{3}$ и $T_{5}$ от параметра $\mu$ степени не вьие 3 и 5 соответственно, такие, что разложение функиии $y_{\mu}(x)$ в ряд Тейлора с точностью до третьего порядка малости задается формулой

$$
y_{\mu}(x)=\mu x+T_{3}(\mu) x^{2}+T_{5}(\mu) x^{3}+\ldots
$$

При этом коэффициент $b_{5}$ при $\mu^{5}$ в полиноме $T_{5}$ связан с коэффиииентом $a_{3}$ при $\mu^{3}$ в полиноме $T_{3}$ соотношением $b_{5}=2 a_{3}^{2}$.

ДокАзАТЕЛЬСтво. Сделав аффинное преобразование образа, можно добиться того, чтобы спрямляющий диффеоморфизм переводил начало координат в себя и имел в этой точке тождественный дифференциал, т. е. чтобы он задавался парой функций $G_{1}(x, y)=x+\ldots, G_{2}(x, y)=y+\ldots$, где отточие обозначает члены второго порядка малости. Кривая $\gamma_{\mu}$ при таком диффеоморфизме переходит в прямую $y=\mu x$, т. е. на кривой $\gamma_{\mu}$ функция $G_{2}(x, y)-\mu G_{1}(x, y)$ обращается в нуль. Лемма 4 получается применением леммы 3 к этой функции.

ТЕОРЕМА О СЕМИ СЕЧЕНИЯХ. Пусть вторая квадратичная форма ростка поверхности в квадрической точке невырожденна. Рассмотрим некоторый пучок сечений поверхности плоскостями, проходящими через квадрическую точку и трансверсальньли $\kappa$ поверхности в этой точке. Допустим, что этот пучок содержит не менее семи сечений. Тогда он локально спрямляется в окрестности квадрической точки, если и только если все секущие плоскости проходят через обшую прямую, трансверсальную поверхности в квадрической точке.

ДокАЗАтЕЛЬство. Если пучок сечений локально спрямляем, то все секущие плоскости $y=\mu x+a z$ пересекают касательную плоскость $z=0$ к поверхности по различным прямым. Поэтому коэффициент $a$ в уравнении сечения является некоторой функцией $g$ от коэффициента $\mu, a=g(\mu)$. Функция $g$ определена для значений параметра $\mu$, соответствующих сечениям из пучка. Если пучок сечений локально спрямляем, то для всех этих значений параметра $\mu$, согласно следствию 1 и лемме 4, должны выполняться следующие соотношения:

(1) $g(\mu) B_{2}(1, \mu)=T_{3}(\mu)$

(2) $g^{2}(\mu)\left(\partial B_{2} / \partial y(1, \mu)\right) B_{2}(1, \mu)+g(\mu) K_{3}(1, \mu)=T_{5}(\mu)$.

Умножив соотношение $(2)$ на $B_{2}$ и воспользовавшись соотношением (1), получим

$$
T_{3}^{2}(1, \mu) \frac{\partial B_{2}}{\partial y}(1, \mu)+T_{3}(\mu) K_{3}(1, \mu)=T_{5}(\mu) B_{2}(1, \mu) .
$$

Если сечений не менее семи, то полиномы, стоящие в левой и правой частях соотношения $(*)$, должны быть тождественно равны: полиномы степени $\leq 7$, имеющие одинаковые старшие коэффициенты, тождественно совпадают, если они совпадают в семи точках. Так как центр пучка является квадрической точкой, то полином $K_{3}(1, \mu)$ делится на полином $B_{2}(1, \mu)$. Так как вторая квадратичная форма в центре пучка невырожденна, то корни полинома $B_{2}$ простые и, следовательно, полином $\left(\partial B_{2} / \partial y\right)(1, \mu)$ не имеет общих корней с полиномом $B_{2}(1, \mu)$. Поэтому из тождества $(*)$ вытекает, что полином $T_{3}^{2}$ делится на полином $B_{2}$. Так как корни полинома $B_{2}$ простые, полином $T_{3}$ должен делиться на полином $B_{2}$. В силу соотношения (1) получаем, что $g(\mu)$ — линейный полином относительно $\mu$. Поэтому все кривые из пучка высекаются на поверхности плоскостями вида $y=\mu x+(p \mu+q) z$, где $p$ и $q$ - некоторые константы. Все эти 
секущие плоскости проходят через общую прямую, трансверсальную поверхности в квадрической точке.

Обратно, пусть все сечения проходят через общую прямую, трансверсальную к поверхности. Тогда параллельное проектирование поверхности вдоль этой прямой спрямляет множество сечений.

ТЕОРемА. Пусть на ростке невьрожденной квадрики в вещественном проективном пространстве задана некоторая риманова метрика, все геодезические относительно которой являются плоскими кривьми. Тогда все плоскости, содержащие геодезические, проходят через одну общую точку, а все семейство геодезических локально спрямляемо.

ДокАЗАТЕЛЬство. Пучок геодезических, выходящих из одной точки риманова многообразия, локально спрямляем: его спрямляет экспоненциальное отображение. Согласно теореме о семи сечениях, плоскости, содержащие геодезические и проходящие через одну точку квадрики, проходят через общую прямую. (Эта теорема применима для геодезических, не касающихся асимптотических направлений, ибо такая кривая не может быть высечена касательной плоскостью. Для геодезических, касательных к асимптотическим направлениям, надо еще воспользоваться непрерывной зависимостью геодезической от направления.) Такие прямые для достаточно близких точек квадрики пересекаются, так как достаточно близкие точки соединяются геодезической. Фиксируем три точки на квадрике, не лежащие на одной геодезической. Соответствующие им три прямые попарно пересекаются и, следовательно, проходят через общую точку, так как эти прямые по условию не лежат в одной плоскости. Прямая, соответствующая любой достаточно близкой точке $A$ на квадрике, тоже проходит через эту общую точку, так как из трех фиксированных точек можно выбрать две, не лежащие с точкой $A$ на одной геодезической. На гладкой поверхности, лежащей в трехмерном пространстве, плоскости, проходящие через фиксированную точку $O$ пространства, высекают двупараметрическое семейство кривых. Это семейство локально спрямляемо около любой точки $A$, отличной от точки $O$, для которой прямая, соединяющая $A$ и $O$, трансверсальна поверхности. Действительно, это семейство спрямляется проектированием из точки $O$.

Итак, для фиксированного ростка квадрики семейство плоских геодезических задается точкой $O$ и зависит, следовательно, от трех параметров. С точностью до проективного преобразования трехмерного пространства таких семейств всего пять: три для эллиптической квадрики (точка $O$ может находиться либо внутри выпуклого тела, ограниченного квадрикой, либо на его границе, либо вне этого тела) и два для гиперболической квадрики (точка $O$ может находиться либо на квадрике, либо вне ее).

3. Метрики. Согласно классической теореме Бельтрами (см. [6, с. 296]), если геодезические на плоской области относительно римановой метрики являются прямыми, то метрика имеет постоянную кривизну. Такая метрика индуцируется при некотором проективном преобразовании либо метрикой модели Клейна геометрии Лобачевского, либо евклидовой метрикой плоскости, либо метрикой Римана на проективной плоскости (здесь имеются в виду классические метрики, определенные с точностью до положительного коэффициента 
пропорциональности, гауссова кривизна для которых может принимать любое вещественное значение). Тем самым мы получаем полное описание римановых метрик на ростке невырожденной квадрики, для которых все геодезические являются плоскими кривыми. Для фиксированного спрямляемого семейства сечений риманова метрика, относительно которой сечения являются геодезическими, зависит от шести параметров: гауссовой кривизнь, которая может принимать любье вещественнье значения, и элемента восьмимерной группь проективных преобразований плоскости, определенного с точностью до правого класса смежности относительно трехмерной подгруппь изометрий соответственно плоскости Лобачевского, плоскости Евклида или плоскости Римана.

СлЕДСТВиЕ 2. (1) Пучок, содержащий не менее семи окружностей или прямых на плоскости, локально спрямляем, если и только если все кривье из него проходят через общую точку, отличную от иентра пучка (см. [5]).

(2) Двупараметрическое семейство прямых и окружностей на плоской области является семейством геодезических относительно некоторой римановой метрики, если и только если это семейство индуцируется при некотором конформном преобразовании плоскости (пополненной бесконечно удаленной точкой) либо семейством геодезических модели Пуанкаре плоскости Лобачевского, либо семейством геодезических плоскости Евклида, либо семейством геодезических плоскости Римана. При этом риманова метрика на плоской области индуцируется соответствующей классической метрикой, определенной с точностью до проективного преобразования.

ДокАЗАТЕЛЬСТВО. При помощи стереографической проекции отобразим плоскость на сферу. При такой проекции прямые и окружности на плоскости перейдут в окружности на сфере, которые являются плоскими сечениями сферы. Следствие теперь вытекает из доказанных выше фактов.

\section{ЛитЕРАТУРА}

1. Хованский Г. С. Основы номографии. Наука, М., 1976. 2. Хованский А. Г., Хованский Г. С. ДАН СССР, 248, № 3, 535-538 (1979). 3. Хованский А. Г., Хованский Г. С. В кн.: Номографический сборник (Хованский Г. С., ред.), № 14, ВТС АН СССР, М., 1982, с. 56-77. 4. Хованский А. Г., Хованский Г. С. В кн.: Номографический сборник (Хованский Г. С., ред.), № 13, ВТС АН СССР, М., 1979, с. 70-105. 5. Хованский А. Г. Сиб. матем. ж., 21, № 4, 221-226 (1980). 6. do Carmo M. P. Differential Geometry of Curves and Surfaces, Prentice-Hall, Englewood Cliffs, NJ, 1976.

Институт системного анализа РАН

Поступило в редакцию 16 декабря 1999 г. 